\title{
Desempeño en ambiente natural de hormigones diseñados para resistir carbonatación
}

\author{
C. Pico-Cortés ${ }^{1 *}$, C. Zega ${ }^{1}$, Y. Villagrán-Zaccardi ${ }^{1,2 * *}$ \\ *carlospicocortes@conicet.gov.ar; **yury.villagranzaccardi@ugent.be \\ ${ }^{1}$ LEMIT, CONICET, La Plata, Argentina \\ ${ }^{2}$ Magnel-Vandepitte Laboratory, UGent, Technologiepark-Zwijnaarde 60, Gent, Bélgica
}

\begin{abstract}
RESUMEN
Se presentan los resultados de carbonatación natural a 17 años de cuatro hormigones diseñados para mitigar el ingreso de $\mathrm{CO}_{2}$. Se emplearon cementos Portland puzolánico y normal, con relaciones agua/cemento (a/c) 0,35, 0,40 y 0,60. El ambiente de exposición fue urbano-industrial, en la ciudad de La Plata, Argentina. Se determinaron la velocidad de carbonatación y su relación con propiedades de transporte evaluadas a 28 días, y se estimó el comportamiento esperado para una vida útil de 50 años. Se identificó una tendencia correlativa lineal con la velocidad de succión capilar, así como menores profundidades de carbonatación asociadas a reducidos valores de propiedades de transporte. Hormigones con cemento puzolánico presentan una ligera mayor velocidad de carbonatación, pero con a/c de 0,40 o menor, verificaron la expectativa de resistir carbonatación en estado de servicio.
\end{abstract}

Palabras clave: Carbonatación natural; propiedades de transporte; velocidad de carbonatación; modelos predictivos; cemento Portland puzolánico.

\section{ABSTRACT}


The 17-year natural carbonation results for four concrete types, designed to mitigate the entry of $\mathrm{CO}_{2}$ are presented. Pozzolanic and ordinary Portland cements were used, with water/cement ratios (w/c) $0.35,0.40$ and 0.60 . The exposure environment was urban-industrial in the city of La Plata, Argentina. The carbonation rate and its correlation with transport properties evaluated at 28 days were determined, and the expected performance for a service life of 50 years was estimated. A linear trend with the capillary absorption rate was identified, with lower carbonation depths for reduced values of transport properties. Concretes with pozzolanic cement demonstrated a slightly higher carbonation rate, but when w/c 0.40 or lower is used, the expected carbonation resistance during service is verified.

Keywords: Natural carbonation; transport properties; carbonation rate; prediction model; pozzolanic Portland cement.

\section{RESUMO}

São apresentados os resultados de 17 anos de carbonatação natural de quatro concretos projetados para mitigar a entrada de $\mathrm{CO}_{2}$. Foram utilizados cimentos Portland pozolânico e normal, com relações água/cimento $(\mathrm{a} / \mathrm{c}):$ 0,35, 0,40 e 0,60. O ambiente de exposição fue urbano-industrial, na cidade de La Plata, Argentina. A taxa de carbonatação e sua relação com as propriedades de transporte avaliadas aos 28 dias foram determinadas, e o comportamento esperado para uma vida útil de 50 anos foi estimado. Identificou-se uma tendência correlativa linear com a velocidade de sucção capilar, bem como menores profundidades de carbonatação associadas a valores reduzidos de propriedades de transporte. Os concretos com cimento puzolânico apresentam uma taxa de carbonatação ligeiramente maior, mas com a/c de 0,40 ou menor, verificaram a expectativa de resistência à carbonatação em estado de serviço.

Palavras chave: Carbonatação natural; propriedades de transporte; taxa de carbonatação; modelos preditivos; cimento Portland pozolânico.

\section{INTRODUCCIÓN}

La carbonatación del hormigón armado es inconveniente para la durabilidad de las estructuras. Su mecanismo es uno de los dos potenciales generadores de procesos corrosivos en las armaduras, junto al ataque por cloruros. La carbonatación ocurre cuando el dióxido de carbono $\left(\mathrm{CO}_{2}\right)$ penetra en el hormigón, fijando y precipitando el calcio y otros álcalis en forma de carbonatos. El avance del frente carbonatado por sobre el recubrimiento y la caída del $\mathrm{pH}$ del hormigón derivan en la despasivación y subsecuente corrosión del acero en presencia de oxígeno y humedad.

\subsection{Vida útil por carbonatación}

La problemática de la corrosión por carbonatación ha llevado a definir la vida útil de estructuras ubicadas en ambientes con concentraciones considerables de $\mathrm{CO}_{2}$ en función de la velocidad de carbonatación natural por parte de algunos reglamentos. Por ejemplo, la Instrucción EHE-08 (Instrucción de Hormigón Estructural EHE, 2008) recomienda utilizar el modelo clásico de Tuutti (Tuutti, 1982) para relacionar la profundidad y velocidad de carbonatación natural con el tiempo de servicio esperado. La ecuación (1) presenta esta relación incluyendo un factor de seguridad propuesto:

$$
x_{c}\left(t_{S L}\right)=K_{\text {field }} \sqrt{t_{d}}=K_{\text {field }} \sqrt{\gamma_{s l} \cdot t_{s l}}
$$


donde $x_{c}$ es la profundidad de carbonatación característica, $t_{d}$ es la vida de servicio de diseño, $t_{s l}$ es el tiempo de servicio que se establece dependiendo del tipo de estructura, $K_{\text {field }}$ es el coeficiente de carbonatación natural, $\gamma_{s l}$ es el factor de seguridad y se puede aproximar a 1,10.

En algunos códigos la vida útil puede incluir alguna parte del período de propagación de la corrosión. Esto se deriva de considerar un estado más avanzado como límite último de servicio.

La correcta estimación de la profundidad de carbonatación requiere de la aplicación simultánea o separada de varias técnicas. Una de estas técnicas se basa en modelos semi-empíricos a partir de las condiciones ambientales de exposición, las propiedades del hormigón endurecido o las características de la mezcla (Loo et al., 1994; Papadakis et al., 1992; Parrott and Killoh, 1989; Possan et al., 2021). Dado que la carbonatación natural require estudios muy prolongados, se han implementado técnicas basadas en datos de carbonatación acelerada. Para su aplicación, algunos autores han establecido diversas formulaciones que relacionan el coeficiente acelerado $(k c)$ con el natural $(k n)$ (Eric I. Moreno, 2013; Neves et al., 2013; Van Den Heede and De Belie, 2014).

Otros autores han planteado la posibilidad de concertar estas dos técnicas combinando coeficientes acelerados con otros parámetros del hormigón para predecir la carbonatación (Yoon et al., 2007). El proceso de comprobar experimentalmente la carbonatación natural es más prolongado, sin embargo es el que otorga mayor fiabilidad debido a que no depende de condiciones simuladas. Por lo tanto, datos de carbonatación natural son aún requeridos para verificar las predicciones mediante modelos (Ekolu, 2018).

La eficacia de los modelos predictivos depende del establecimiento de correlaciones apropiadas con la velocidad de carbonatación natural. Por ejemplo, en el modelo de (Parrott, 1994) se incluyó a la permeabilidad al oxígeno para estimar la velocidad de carbonatación. Las propiedades de transporte presentan mayor correlación con la carbonatación natural que las propiedades mecánicas; incluyendo el caso de hormigones sustentables como hormigón reciclado o con materiales cementíceos suplementarios (MCS) (Kurda et al., 2019; Zega et al., 2019). En estos casos, parámetros como la absorción capilar, la porosidad total y la penetración de ion cloruro a edades entre 28 días y 1 año han presentado las mayores eficiencias para predecir la carbonatación. Las condiciones climáticas pueden tenerse en cuenta en la predicción de carbonatación natural. En general, los modelos predictivos utilizan valores de $\mathrm{HR}$, concentración de $\mathrm{CO} 2$ y/o precipitaciones promedio. Sin embargo, las variaciones en el tiempo de estos factores pueden influir notoriamente (Visser, 2014). También influyen los ciclos mojado - secado y el aumento de temperatura, causando incremento de la difusión de $\mathrm{CO}_{2}$ (Chen et al., 2018).

\subsection{Medición de la carbonatación natural}

Los ensayos de carbonatación natural de hormigón conservan condiciones de exposición similares a las que eventualmente tendrían estructuras construidas con el material. De allí la importancia de obtener datos experimentales con condiciones representativas.

En (Eric Ivan Moreno et al., 2016) se presentaron resultados parciales de carbonatación natural en México y España. Los resultados a 24 meses indicaron que los coeficientes de carbonatación fueron mayores para ambientes con menor humedad relativa (HR), en un rango entre el $80 \%$ y el 100\%. Esto en consecuencia de una mayor aproximación al rango de 50\% a 60\%, aceptado como aquel que presenta máxima carbonatación natural (Galán, 2011; Wierig, 1984). Para un período de exposición similar (2,5 años), se encontró que el contenido de $\mathrm{CaO}$ en el cemento y particularmente la relación agua/CaO reactivo es otro factor influyente que aumenta la velocidad de carbonatación (Leemann et al., 2015).

\subsection{Materiales cementantes y carbonatación}

La carbonatación no solo depende de las condiciones ambientales sino también del tipo de cemento. 
Hormigones con cemento Portland sin MCS han presentado, en general, menores velocidades de carbonatación (Sanjuán et al., 2003). El alto contenido de clínker se asocia frecuentemente a una alta resistencia al ingreso de sustancias agresivas al hormigón.

Los hormigones con MCS presentan, en general, mayor velocidad de carbonatación natural. Su evaluación requiere la consideración de propiedades a edades avanzadas debido a los plazos que requieren las reacciones puzolánicas.

Desde hace más de 50 años se viene experimentando la carbonatación natural de hormigones adicionados. Ya en 1986, Nagataki et al. experimentaron con hormigones con ceniza volante al 30\% de reemplazo de cemento fabricados en 1969 (Nagataki et al., 1986). Se determinó la carbonatación natural a 2, 5, 10 y 15 años. Los autores encontraron que después de 5 años la velocidad de carbonatación comenzó a reducirse para hormigones exteriores. En cambio, continuó aumentando para hormigones interiores, los cuales inicialmente presentaban menor aceleración.

Recientemente, cementantes alternativos del cemento Portland normal han presentado mayor variabilidad en su velocidad de carbonatación natural (Lollini and Redaelli, 2021; Ribeiro et al., 2018).

En (Ribeiro et al., 2018) se evaluó la carbonatación natural de hormigones con ceniza volante (CV) y escoria de alto horno (EAH) durante 10 años. Se encontró que el valor $k$ de durabilidad fue mayor para los hormigones con EAH, debido a su menor velocidad de carbonatación. Sin embargo, las profundidades de carbonatación fueron menores a $5 \mathrm{~mm}$ para todos los casos, lo cual reflejó la necesidad de evaluación por desempeño por encima de un enfoque prescriptivo.

La selección del tipo de cemento base es importante debido a la potencial incidencia de la reacción puzolánica (Rabehi et al., 2013). Algunos MCS inertes como el filler calcáreo pueden favorecer a la carbonatación más que aquellos que consumen hidróxido de calcio (Lollini and Redaelli, 2021). El consumo de portlandita rige como factor principal debido a la reducción de la reserva alcalina, pero también reduce la conectividad de poros y aumenta la tortuosidad. Por lo tanto, puede producirse un desfasaje en la correlación entre la velocidad de carbonatación y otras propiedades de transporte en los hormigones adicionados, en relación a hormigón con cemento Pórtland normal. En (von Greve-Dierfeld et al., 2020) se encontró que el consumo de hidróxido de calcio de los MCS puede causar aumento de la velocidad de carbonatación debido a la disminución de la reserva alcalina. En consecuencia, se provocaría una caída más rápida del $\mathrm{pH}$. En este sentido, el cemento Portland normal mantiene ciertas ventajas para resistir a la carbonatación.

La variabilidad de los componentes, proporciones y características de las mezclas de hormigón influyen en dos factores principales para la resistencia a la carbonatación. Primero, el factor físico determinado por la porosidad y la velocidad de difusión del $\mathrm{CO}_{2}$. Y segundo, el factor químico, relacionado con la reserva alcalina de la pasta, que es distinta para cada tipo de cemento. La naturaleza de las reacciones, el consumo de portlandita y los productos derivados de la carbonatación influyen en el factor químico.

Este trabajo presenta los resultados de carbonatación natural de hormigones con cemento portland normal y hormigones con cemento Portland puzolánico. Todos han sido diseñados con el fin de resistir carbonatación y han sido expuestos al ambiente natural durante 17 años.

\section{MATERIALES Y PROCEDIMIENTO}

\subsection{Componentes y mezclas}

Fueron utilizados tres tipos de cemento Portland con resistencia nominal de tipo CP40, uno de tipo Normal (CPN) y dos de tipo Puzolánico (CPP-1 y CPP-2). Se fabricaron mezclas con agregado fino silíceo natural y agregado grueso de piedra partida granítica de tamaño nominal 6-20mm. Las relaciones a/c utilizadas fueron 0,$35 ; 0,40$ y 0,60 . Además se incluyeron aditivos de lignosulfonato 
modificado como plastificante y sulfonato de naftaleno como superplastificante en las mezclas con a/c de 0,35 . Éstos permitieron reducir la cantidad de agua procurando una red de poros más cerrada sin detrimento de la fluidez. En la Tabla 1 se presenta la caracterización física y química de los cementos, en la Tabla 2 las propiedades de los agregados y en la Tabla 3 las características de las mezclas.

Tabla 1. Caracterización física y química de los cementos.

\begin{tabular}{|c|c|c|c|}
\hline Composición química & $\mathbf{C P N}$ & CPP-1 & CPP-2 \\
\hline $\mathrm{SiO}_{2}(\%)$ & 19,86 & 14,79 & 16,83 \\
\hline $\mathrm{CaO}(\%)$ & 63,82 & 50,32 & 48,94 \\
\hline $\mathrm{Fe}_{2} \mathrm{O}_{3}(\%)$ & 4,19 & 3,09 & 3,97 \\
\hline $\mathrm{Al}_{2} \mathrm{O}_{4}(\%)$ & 4,11 & 4,77 & 4,46 \\
\hline $\mathrm{Na}_{2} \mathrm{O}(\%)$ & 0,01 & 0,16 & 0,12 \\
\hline $\mathrm{K}_{2} \mathrm{O}(\%)$ & 0,93 & 1,24 & 1,15 \\
\hline $\mathrm{MgO}(\%)$ & 1,60 & 1,01 & 1,64 \\
\hline $\mathrm{SO}_{3}(\%)$ & 1,73 & 1,40 & 1,84 \\
\hline $\mathrm{Cl}^{-}(\%)$ & 0,03 & 0,03 & 0,02 \\
\hline Pérdida por calcinación $(\%)$ & 0,87 & 1,20 & 5,36 \\
\hline Residuo Insoluble (\%) & 2,65 & 21,65 & 14,90 \\
\hline \multicolumn{4}{|l|}{ Caracterización física } \\
\hline Superficie Específica Blaine $\left(\mathrm{m}^{2} / \mathrm{kg}\right)$ & 288 & 281 & 416 \\
\hline Retenido en tamiz $75 \mu \mathrm{m}(\%)$ & 2,4 & 2,1 & 10,4 \\
\hline Densidad $\left(\mathrm{g} / \mathrm{cm}^{3}\right)$ & 3,13 & 2,95 & 2,99 \\
\hline Resistencia a la compresión $2 \mathrm{~d}$ & 20,4 & 18,9 & 10,5 \\
\hline Resistencia a la compresión $28 \mathrm{~d}$ & 44,4 & 41,8 & 40,1 \\
\hline
\end{tabular}

Tabla 2. Propiedades de los agregados.

\begin{tabular}{|l|c|c|}
\hline \multirow{2}{*}{\multicolumn{1}{|c|}{ Propiedad }} & \multicolumn{2}{c|}{ Agregados } \\
\cline { 2 - 3 } & Fino & Grueso \\
\hline Módulo de Finura & 2,30 & 6,71 \\
\hline Tamaño máximo (mm) & nd & 19 \\
\hline Densidad saturada y superficie seca & 2,67 & 2,75 \\
\hline Absorción de agua (\%) & 0,5 & 0,2 \\
\hline
\end{tabular}

Las propiedades medidas en estado fresco fueron: asentamiento a través del ensayo del cono de Abrams según ASTM C143, aire incorporado por el método de presión según ASTM C231 y porcentaje de exudación según ASTM C232. 
Tabla 3. Materiales y propiedades en estado fresco de los hormigones.

\begin{tabular}{|l|c|c|c|c|}
\hline Materiales & N35 & P35 & P40 & P60 \\
\hline Relación a/c & 0,35 & 0,35 & 0,40 & 0,60 \\
\hline CPN & 400 & - & - & - \\
\hline CPP-1 & - & 400 & - & - \\
\hline CPP-2 & - & - & 442 & 290 \\
\hline Agregado fino & 927 & 895 & 800 & 929 \\
\hline Agregado grueso & 979 & 979 & 981 & 992 \\
\hline Plastificante & 3,2 & 3,2 & - & - \\
\hline Superplastificante & 4,0 & 5,6 & - & - \\
\hline Propiedades en estado fresco & & & & \\
\hline Asentamiento (mm) & 110 & 150 & 45 & 50 \\
\hline Aire Incorporado (\%) & 4,2 & 3,2 & 1,7 & 1,9 \\
\hline Exudación (\%) & $<0,01$ & $<0,01$ & nd & nd \\
\hline
\end{tabular}

Con cada una de las mezclas se moldearon probetas prismáticas de $75 \mathrm{mmx} 75 \mathrm{~mm} \times 300 \mathrm{~mm}$ para ser sometidas a carbonatación natural, probetas normalizadas cilíndricas para resistencia a compresión, probetas cúbicas de $200 \mathrm{~mm}$ de arista para evaluar permeabilidad a través de la penetración de agua a presión y probetas cilíndricas de $100 \mathrm{~mm} \times 200 \mathrm{~mm}$ para ensayos de absorción de agua, succión capilar y migración de ion cloruro. Todas las muestras fueron desmoldadas a las 24 horas.

\subsection{Curado y exposición}

Todas las muestras de hormigón fueron curadas durante 28 días en condiciones normalizadas (T: $23 \pm 2^{\circ} \mathrm{C}$; HR $>95 \%$ ). Posteriormente las muestras para carbonatación fueron expuestas a la acción ambiental del $\mathrm{CO}_{2}$ en una zona urbana-industrial sin contacto con el suelo.

Las condiciones de exposición natural fueron en la ciudad de La Plata, Argentina, con humedad relativa promedio variable entre $70 \%$ y $83 \%$, según se presenta en la Figura 1, temperaturas entre $5,5^{\circ} \mathrm{C}$ (mes de julio) y $28,8^{\circ} \mathrm{C}$ (mes de enero), precipitaciones entre $54,6 \mathrm{~mm}$ (mes de junio) y $117,9 \mathrm{~mm}$ (mes de marzo) con 8 días de lluvia en promedio por mes, como se observa en la Figura 2. La concentración de $\mathrm{CO}_{2}$ varió entre 400 y 580 ppm para el período de exposición.

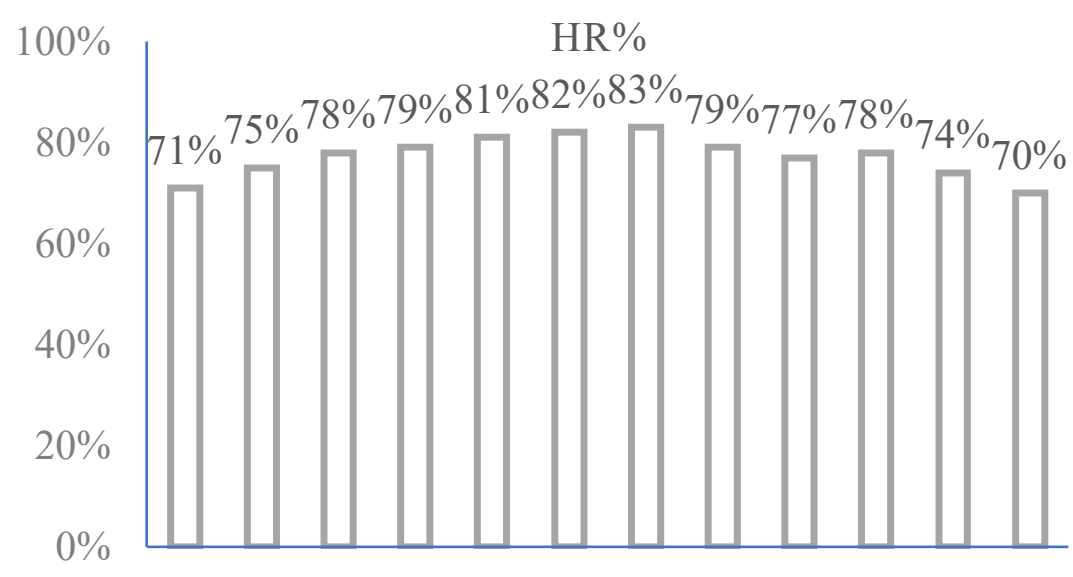

Ene Feb Mar Abr May Jun Jul Ago Sep Oct Nov Dic

Figura 1. Humedad relativa mensual promedio en La Plata, Argentina - Datos históricos de 50 años. Fuente: Servicio Meteorológico Nacional 


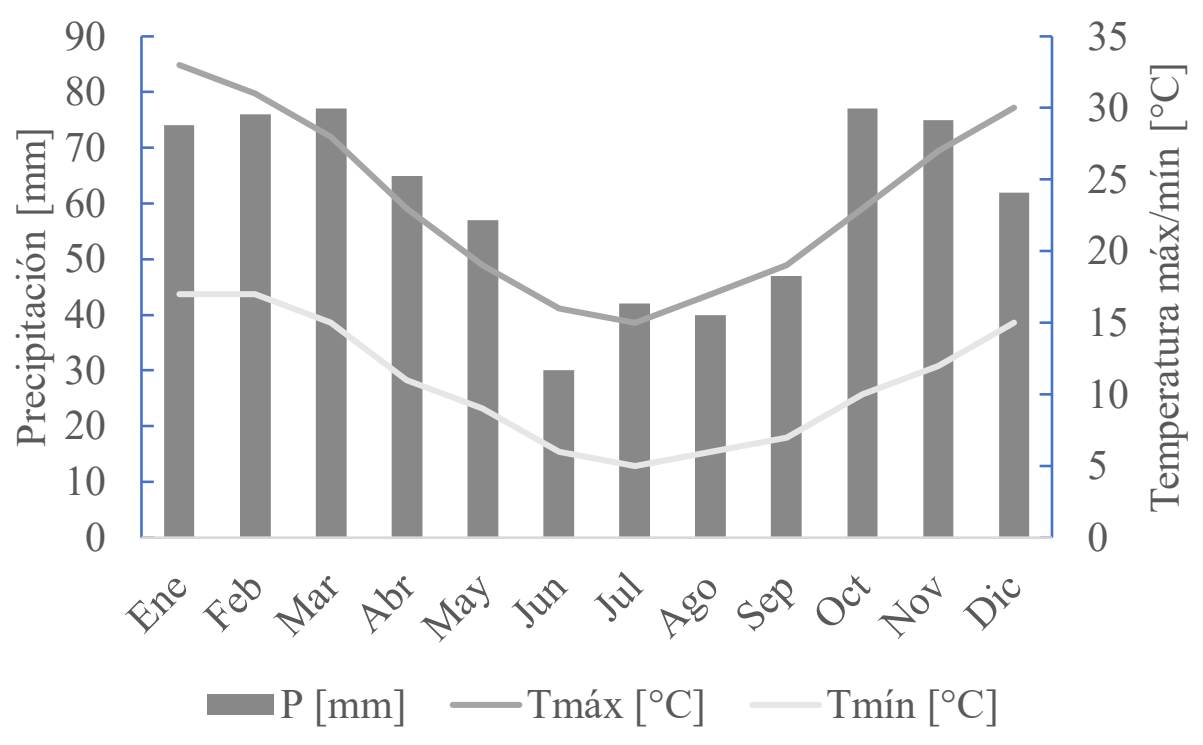

Figura 2. Media de precipitaciones y de temperatura máximas y mínimas en La Plata, Argentina Datos históricos de 30 años. Fuente: Servicio Meteorológico NEMS

\subsection{Medición}

Pasado el tiempo de exposición, se realizó la medición del espesor de carbonatación. Se aserró una rodaja de cada una de las probetas, rociándola luego con la solución alcohólica de fenolftaleína al $1 \%$ y se midió la profundidad del frente carbonatado en los cuatro lados. Los ensayos complementarios fueron realizados de acuerdo a normas de la siguiente manera: resistencia a la compresión según ASTM C 39/C 39M, absorción de agua y porosidad según ASTM C 642, penetración de agua a presión según IRAM 1554, succión capilar según IRAM 1871 y migración de ion cloruro según NT Build 492.

Por último, y con el fin de determinar la vida útil por carbonatación de una estructura de 50 años, se compararon los resultados obtenidos en la exposición natural con los resultados de la predicción de profundidad de carbonatación a través del modelo clásico planteado por (Papadakis et al., 1992). Este modelo fue diseñado inicialmente para hormigón de CPN y se distingue por su practicidad para la estimación a partir de los constituyentes del hormigón. Es descrito en la ecuación (2)

$$
x_{c t}=\left(\frac{2\left[\mathrm{CO}_{2}\right]^{0} D_{e, \mathrm{CO}_{2}}}{[\mathrm{CH}]+3[\mathrm{CSH}]} t\right)^{1 / 2}
$$

donde la profundidad de carbonatación $x_{c t}$ está en $\mathrm{m},\left[\mathrm{CO}_{2}\right]^{0}$ es la concentración molar de $\mathrm{CO}_{2}$ ambiental en $\mathrm{mol} / \mathrm{m}^{3}, D_{e, C O}$ es la difusividad efectiva de $\mathrm{CO}_{2}$ en el hormigón en $\mathrm{m}^{2} /$ año, $[\mathrm{CH}]+3[\mathrm{CSH}]$ es la concentración molar total de constituyentes carbonatables del hormigón en $\mathrm{mol} / \mathrm{m}^{3}, t$ es el tiempo en años, y $H R$ es el valor medio de la humedad relativa. La concentración molar del carbono ambiental se puede calcular según la ecuación (3), donde $y_{\mathrm{CO}_{2}}$ es el contenido ambiental de $\mathrm{CO}_{2}$ por volumen en ppmv. La difusividad efectiva del $\mathrm{CO}_{2}$ en el hormigón se calcula según la ecuación (4), donde $\varepsilon_{p}$ es la porosidad de los componentes completamente hidratados y carbonatados de la pasta de cemento, estimada según la ecuación (5). La concentración molar total de los constituyentes carbonatables del hormigón pueden estimarse según la ecuación (6). 


$$
\begin{aligned}
& {\left[\mathrm{CO}_{2}\right]^{0}=42 y_{\mathrm{CO}_{2}} \cdot 10^{-6}} \\
& D_{e, C O_{2}}=51.8 \varepsilon_{p}^{1.8}\left[1-\left(\frac{H R}{100}\right)\right]^{2.2} \\
& \varepsilon_{p} \approx\left(\frac{\rho_{c}}{\rho_{a}}\right) \frac{a / c-0.3}{1+\left(\rho_{c} / \rho_{a}\right)(a / c)} \\
& {[C H]+3[C S H] \approx \frac{1+\left(\rho_{c} / \rho_{a}\right)(a / c)+\left(\rho_{c} / \rho_{a g}\right)(a g / c)}{33000}}
\end{aligned}
$$

En las ecuaciones (5) y (6) $\rho_{c}, \rho_{a}$ y $\rho_{a g}$ son las densidades de cemento, agua, y agregados en $\mathrm{kg} / \mathrm{m}^{3}$, respectivamente; y $c, a, \mathrm{y} a g$ son los contenidos de cemento, agua y agregados en $\mathrm{kg} / \mathrm{m}^{3}$, respectivamente.

\section{RESULTADOS Y DISCUSIÓN}

En la Tabla 4 se presentan los resultados de propiedades complementarias para cada tipo de hormigón determinadas a 28 días de edad.

Tabla 4. Propiedades complementarias a la carbonatación.

\begin{tabular}{|l|c|c|c|c|}
\hline Propiedades en estado endurecido & N35 & P35 & P40 & P60 \\
\hline Resistencia a la compresión 28d (MPa) & 58,20 & 57,40 & 31,75 & 23,10 \\
\hline Absorción de agua 24h $(\%)$ & $2,50 \%$ & $2,90 \%$ & $5,00 \%$ & $5,15 \%$ \\
\hline Porosidad (\%) & $6,00 \%$ & $6,85 \%$ & $11,50 \%$ & $11,80 \%$ \\
\hline Penetración de agua a presión máx $(\mathrm{mm})$ & 32 & 28 & 48 & 57 \\
\hline Velocidad de succión capilar $\left(\mathrm{g} \cdot \mathrm{m}^{-2} \cdot \mathrm{s}^{-0.5}\right)$ & 0,95 & 1,15 & 1,60 & 2,90 \\
\hline Coef. Migración de ion cloruro $\mathrm{m}^{2} / \mathrm{s}$ & $4,6 \times 10^{-12}$ & $3,3 \times 10^{-12}$ & $4,9 \times 10^{-12}$ & $14 \times 10^{-12}$ \\
\hline
\end{tabular}

El espesor de carbonatación natural se midió luego de 14 y 17 años de exposición, como se observa en la Figura 3. Para los tipos de hormigón N35 y P35 el avance fue prácticamente el mismo para ambas edades. La velocidad de carbonatación fue mayor para las muestras de P40 y P60. 


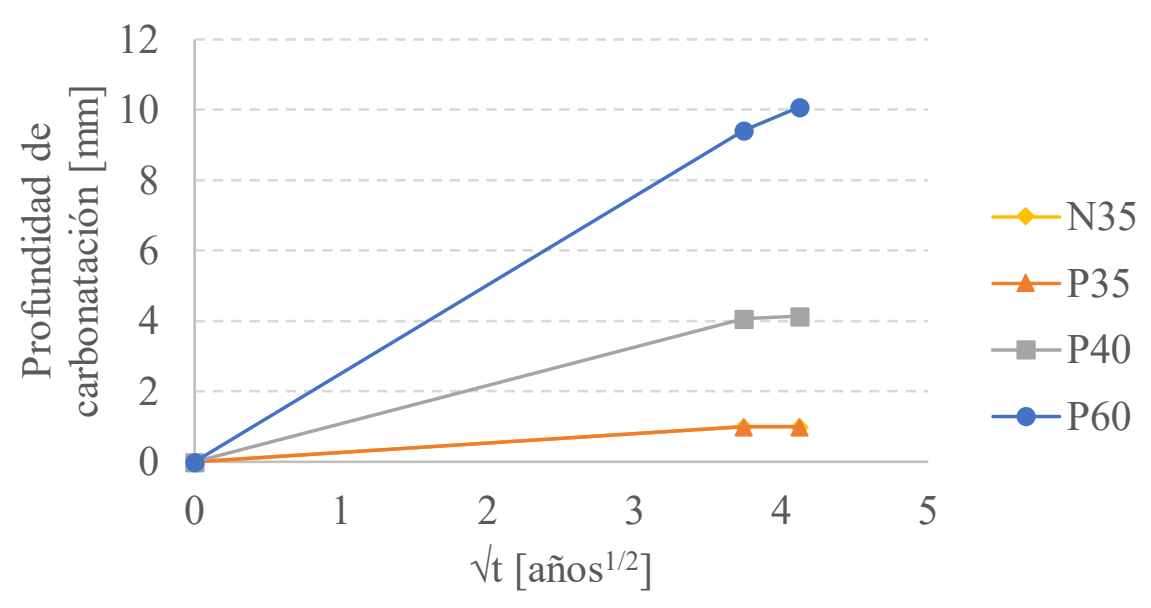

Figura 3. Evolución de la profundidad de carbonatación con la raíz del tiempo.

Con la relación entre profundidad de carbonatación y raíz del tiempo es posible encontrar el coeficiente de velocidad de carbonatación, según la ecuación (1) (Tuutti, 1982). El resultado se presenta en la Tabla 5.

Tabla 5. Profundidad de carbonatación medida a 17 años y velocidad de carbonatación obtenida por el modelo de Tuutti

\begin{tabular}{|c|c|c|c|c|}
\hline Tipo de Hormigón & N35 & P35 & P40 & P60 \\
\hline $\begin{array}{c}\text { Profundidad de carbonatación } \\
\text { promedio a 17 años (mm) }\end{array}$ & 0,95 & 0,97 & 4,14 & 10,08 \\
\hline $\begin{array}{c}\text { Velocidad de carbonatación } \\
K_{\text {field }}\left(\mathrm{mm} \cdot \text { años }^{-0,5}\right)\end{array}$ & 0,23 & 0,235 & 1,004 & 2,445 \\
\hline
\end{tabular}

Se encontró una buena correlación entre la velocidad de carbonatación $K_{\text {field }} \mathrm{y}$ algunas de las propiedades complementarias. En las Figuras 4 a 6 se presentan las más significativas, $\mathrm{R}^{2}$ mayor a 0,98. Como es de esperar, para el caso de la resistencia a la compresión se encontró una relación inversa, como se observa en la Figura 4.

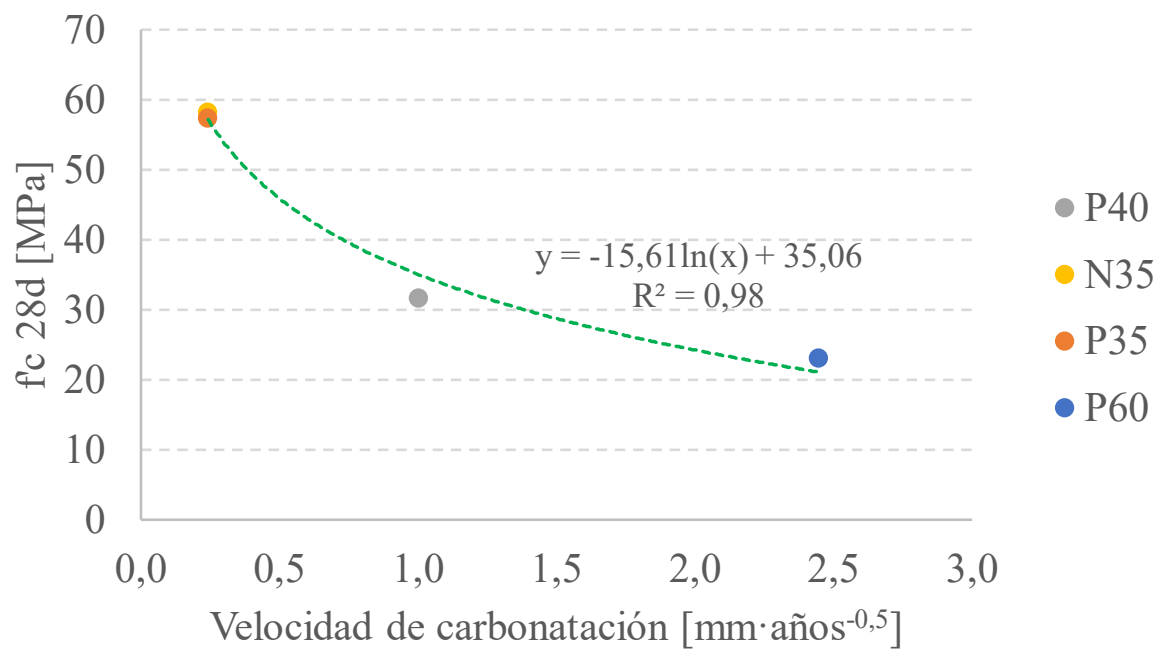

Figura 4. Resistencia a la compresión f' c a 28 días versus velocidad de carbonatación $K_{\text {field }}$. 
En relación a la penetración de agua a presión máxima, la línea de tendencia presentó un comportamiento logarítmico positivo, descrito en la Figura 5. Mientras que para la velocidad de succión capilar se presentó una tendencia lineal positiva, según se observa en la Figura 6.

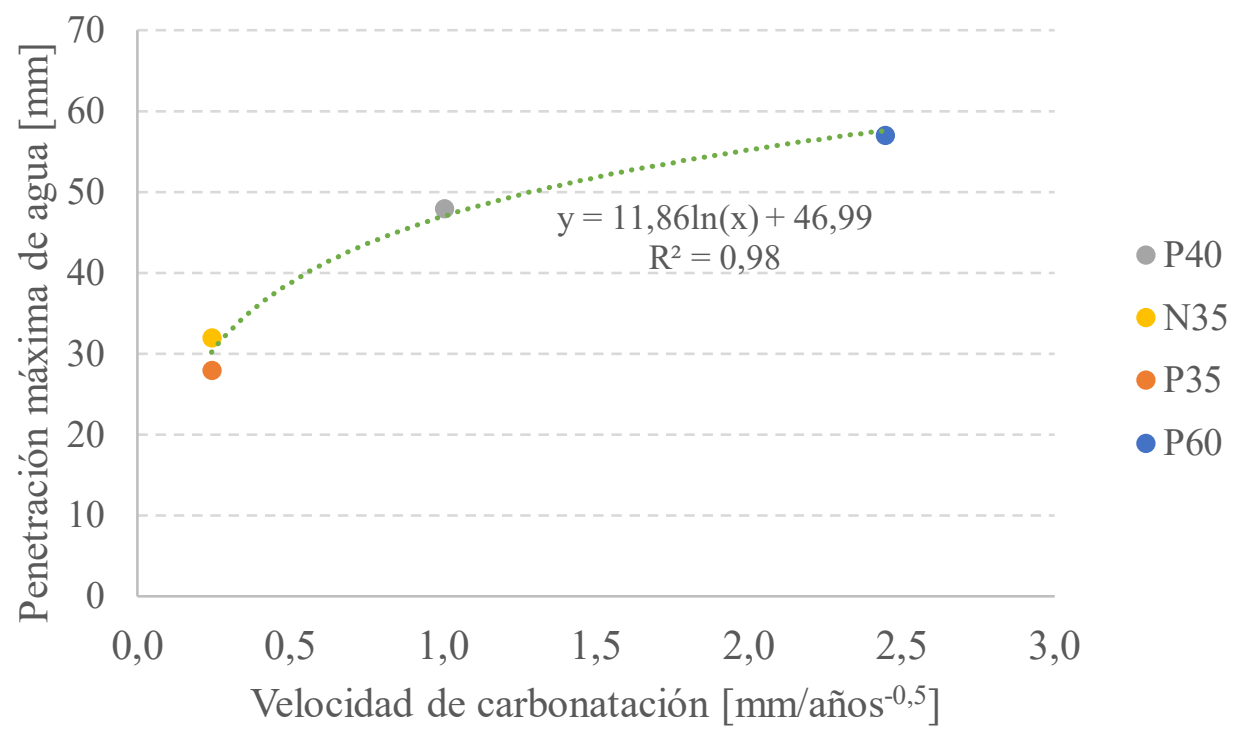

Figura 5. Penetración de agua a presión a 28 días versus velocidad de carbonatación $K_{\text {field }}$.

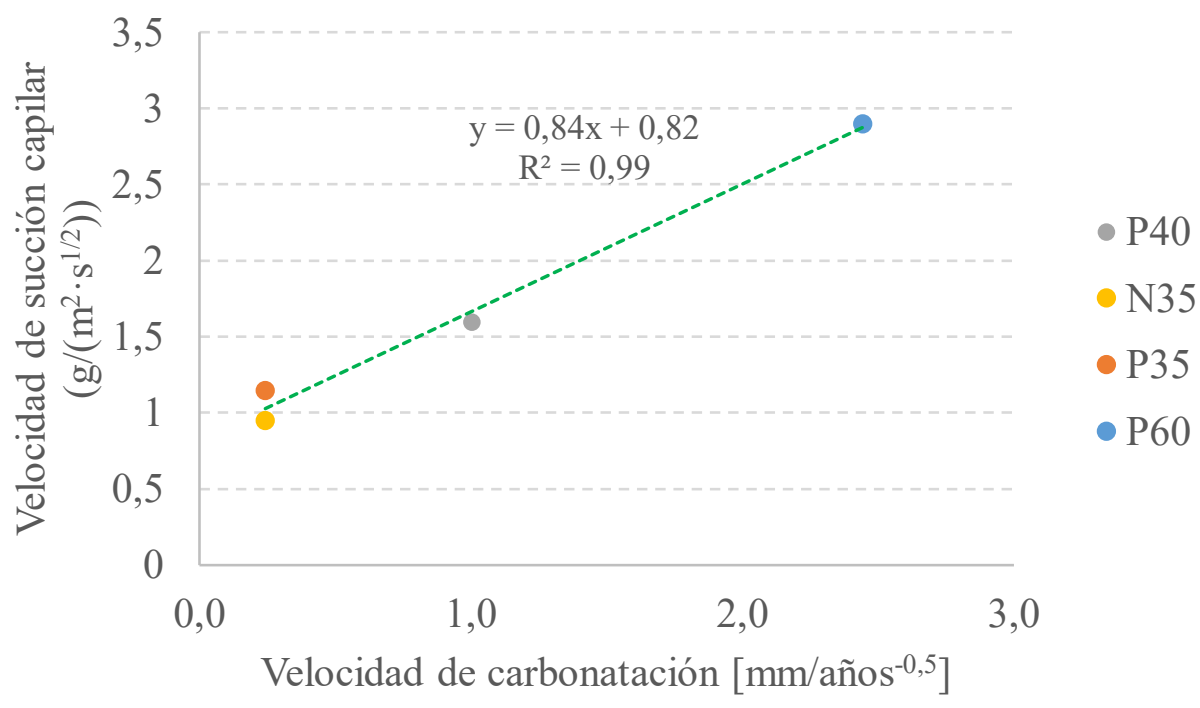

Figura 6. Velocidad de succión capilar a 28 días versus velocidad de carbonatación $K_{\text {field }}$.

La mayor de las velocidades de succión capilar presentadas para este grupo de hormigones fue de $2,90 \mathrm{~g} /\left(\mathrm{m}^{2} \cdot \mathrm{s}^{1 / 2}\right)$, para P60.

El modelo predictivo simplificado de (Papadakis et al., 1992) arrojó los resultados mostrados en la Tabla 6 para cada uno de sus parámetros aplicados a los cuatro tipos de hormigón.

Tabla 6. Estimación de la profundidad de carbonatación con el modelo de Papadakis.

\begin{tabular}{|c|c|c|c|c|}
\hline Tipo de Hormigón & N35 & P35 & P40 & P60 \\
\hline$\left[\mathrm{CO}_{2}\right]^{0}\left(\mathrm{~mol} / \mathrm{m}^{3}\right)$ & 0,0206 & 0,0206 & 0,0206 & 0,0206 \\
\hline
\end{tabular}




\begin{tabular}{|l|c|c|c|c|}
\hline$\varepsilon_{p}(\%)$ & $7,47 \%$ & $7,26 \%$ & $13,62 \%$ & $32,10 \%$ \\
\hline$D_{e, C O 2}\left(\mathrm{~m}^{2} / \mathrm{año}\right)$ & 0,0187 & 0,0177 & 0,0551 & 0,2579 \\
\hline$[\mathrm{CH}]+3[\mathrm{CSH}]\left(\mathrm{mol} / \mathrm{m}^{3}\right)$ & 4344 & 4629 & 4974 & 3268 \\
\hline$t$ (años) & 50 & 50 & 50 & 50 \\
\hline$R H$ promedio $(\%)$ & $77,25 \%$ & $77,25 \%$ & $77,25 \%$ & $77,25 \%$ \\
\hline $\begin{array}{l}\text { Profundidad de carbonatación } \\
\text { a 50 años, } \mathrm{x}_{\mathrm{ct}}(\mathrm{mm})\end{array}$ & 3,0 & 2,8 & 4,8 & 12,7 \\
\hline
\end{tabular}

La influencia de la relación a/c y del contenido de cemento en la porosidad teórica (ecuación 5) según el modelo de Papadakis es amplia. Puede deducirse de la Tabla 6 que los valores de profundidad de carbonatación esperados para P60 son 2,65 veces mayores que los valores esperados para P40, 4,2 veces que para N35 y 4,5 veces que para P35. Al comparar el porcentaje de porosidad teórico $\left(\varepsilon_{p}\right)$ de la ecuación (5) con el determinado experimentalmente en la Tabla 4, se observa que para N35, P35 y P40 existen ligeras sobreestimaciones, mientras que para P60 la sobreestimación es muy significativa (más del triple del valor medido experimentalmente). El valor de $\varepsilon_{p}$ en la Tabla 6 no puede considerarse esperado para un hormigón con a/c $=0,60$, por lo que debe considerarse con escepticismo. El modelo simplificado de Papadakis está inicialmente diseñado para hormigones sin MCS y no tiene en cuenta la inclusión de otras variables como los aditivos. En consecuencia, puede predecir valores distintos a los experimentales en hormigones modernos. Sin embargo, es un punto de partida interesante debido a la comparabilidad que provee entre modelos predictivos basados en variables influyentes y el diseño por desempeño. El modelo de Tuutti puede ser más adecuado para estructuras en servicio, siempre y cuando se utilicen factores de seguridad adecuados basados en la porosidad. En la Tabla 7 se presentan los resultados de la profundidad de carbonatación para una vida útil de 50 años estimada con la ecuación (1) y los coeficientes de carbonatación determinados en campo. Puede verse que los hormigones N35 y P35 podrían considerarse ultra durables en términos de la carbonatación, siempre que se prevenga la fisuración del recubrimiento por razones estructurales, de servicio o de durabilidad. Además, se puede comprobar que el modelo simplificado de Papadakis tiene una correlación limitada con la carbonatación natural de los hormigones estudiados. El modelo provee un enfoque más liberal para P40 y P60 que el determinado por desempeño y un enfoque más conservador para N35 y P35. Es sorpresivo que, a pesar de la excesiva sobreestimación de $\varepsilon_{p}$ en el modelo de Papadakis, la estimación del espesor carbonatado para P60 resulta menor que la determinada según el modelo de Tuutti. Por lo tanto, la relación entre la porosidad y difusividad de $\mathrm{CO}_{2}$ necesitaría ser reconsiderada para mejorar estas predicciones y su aplicación en hormigones con MCS.

Tabla 7. Profundidad de carbonatación para vida útil supuesta de 50 años según EHE-08.

\begin{tabular}{|l|c|c|c|c|}
\hline Tipo de Hormigón & N35 & P35 & P40 & P60 \\
\hline $\begin{array}{l}\text { Profundidad de carbonatación proyectada para } \\
\text { vida útil supuesta de 50 años, } \mathrm{x}_{\mathrm{c}}(\mathrm{mm})\end{array}$ & 1,7 & 1,7 & 7,4 & 18,1 \\
\hline
\end{tabular}

Las profundidades de carbonatación determinadas a partir de estimaciones de desempeño y las velocidades de succión capilar presentadas en la Figura 6 pueden correlacionarse. Se observa que todas las velocidades de succión capilar son menores al límite de $4,0 \mathrm{~g} /\left(\mathrm{m}^{2} \cdot \mathrm{s}^{1 / 2}\right)$, sugerido en el Reglamento Argentino de Estructuras de Hormigón (Instituto Nacional de Tecnología Industrial, 2005). Este límite procura la durabilidad para estructuras de hormigón armado a 50 años, por lo que deberá verificarse que la carbonatación natural no supere espesores de recubrimiento típicos. 
$\geq 0,60$ podría ser propuesta.

\section{CONCLUSIONES}

Se presentan resultados de carbonatación natural a 17 años para hormigones con CPN y CPP. Para las mezclas con relación a/c de 0,35 y aditivo los valores de profundidad de carbonatación luego de 17 años fueron de aproximadamente $1 \mathrm{~mm}$, similar medición a la realizada a 14 años. Los espesores carbonatados obtenidos para estos hormigones son demasiado bajos como para tener una determinación precisa de la velocidad de carbonatación, por lo que deben seguirse evaluando. Aun así, dichos hormigones pueden ser considerados ultra durables frente a la carbonatación. El factor químico no resultó de gran incidencia en estos hormigones, ya que la carbonatación se vio limitada por la muy reducida estructura de poros. Para los hormigones P40 y P60 la carbonatación se hace más notoria conforme se incrementa $\mathrm{a} / \mathrm{c}$.

La velocidad de carbonatación natural calculada con la profundidad sobre la raíz cuadrada del tiempo a 17 años presentó correlación lineal con la velocidad de succión capilar calculada a 28 días de edad. La velocidad de succión capilar se presenta como uno de los índices más adecuados para predecir la carbonatación. La linealidad presentada en este trabajo entre velocidad de carbonatación natural y velocidad de succión capilar a 28 días puede contribuir a evaluaciones futuras sobre esta correlación. En caso de comprobarse, y respecto al énfasis prescriptivo, las velocidades de carbonatación críticas podrían determinar los límites del coeficiente de succión capilar para estructuras sometidas a carbonatación, sobre todo para hormigones con relaciones a/c mayores o iguales a 0,60 .

La predicción de profundidad de carbonatación a través del modelo simplificado de Papadakis provee idea del comportamiento del hormigón a partir de sus constituyentes. Esta es una ventaja desde el punto de vista práctico. Sin embargo, el modelo parece requerir algunas revisiones en cuanto a la estimación de la porosidad, la difusividad de $\mathrm{CO}_{2}$ y la consideración del efecto de MCS, entre otras variables.

\section{AGRADECIMIENTOS}

Los autores agradecen al Fondo para la Investigación Científica y Tecnológica de la República Argentina a través del PICT-2017 0091. Yury Villagrán-Zaccardi agradece a Research FoundationFlanders (FWO-Vlaanderen) por el financiamiento a través del Proyecto 12ZZD21N Marie Skłodowska-Curie Actions - Seal of Excellence.

\section{REFERENCIAS}

Chen, Y., Liu, P. and Yu, Z. (2018). Effects of environmental factors on concrete carbonation depth and compressive strength. Materials. 11(2167):1-11.

https://doi.org/10.3390/ma11112167

Comisión Permanente del Hormigón, EHE (2008), “Instrucción de Hormigón Estructural. Ministério de obras públicas e urbanismo". Madrid, España.

Ekolu, S. O. (2018). Model for practical prediction of natural carbonation in reinforced concrete: Part 1-formulation. Cement and Concrete Composites. 86:40-56. https://doi.org/10.1016/j.cemconcomp.2017.10.006

Galán, I. (2011). Carbonatación del hormigón: combinación de CO2 con las fases hidratadas del cemento $y$ frente de cambio de $\mathrm{pH}$, Tesis de Doctorado, Universidad Complutense de 
Madrid, 202 p. https://core.ac.uk/download/pdf/19716738.pdf

Instituto Nacional de Tecnología Industrial. (2005). CIRSOC-201 Reglamento Argentino de Estructuras de Hormigón Armado, Buenos Aires, Argentina.

Kurda, R., De Brito, J. and Silvestre, J. D. (2019). Carbonation of concrete made with high amount of fly ash and recycled concrete aggregates for utilization of $\mathrm{CO} 2$. Journal of CO2 Utilization. 29:12-19. https://doi.org/10.1016/j.jcou.2018.11.004

Leemann, A., Nygaard, P., Kaufmann, J. and Loser, R. (2015). Relation between carbonation resistance, mix design and exposure of mortar and concrete. Cement and Concrete Composites. 62:33-43. https://doi.org/10.1016/j.cemconcomp.2015.04.020

Lollini, F. and Redaelli, E. (2021). Carbonation of blended cement concretes after 12 years of natural exposure. Construction and Building Materials. 276:122-131. https://doi.org/10.1016/j.conbuildmat.2020.122122

Loo, Y. H., Chin, M. S., Tam, C. T. and Ong, K. C. G. (1994). A carbonation prediction model for accelerated carbonation testing of concrete. Magazine of Concrete Research. 46 (168):191-200. https://doi.org/10.1680/macr.1994.46.168.191

Moreno, Eric I. (2013). Carbonation coefficients from concrete made with high-absorption limestone aggregate. Advances in Materials Science and Engineering. 734031:1-4 https://doi.org/10.1155/2013/734031

Moreno, Eric Ivan, Torres-Acosta, A., Pérez-Quiroz, J. T., Martínez-Madrid, M., MartínezMolina, W., Alonso-Guzmán, E., Castro-Borges, P., Genescá-Llongueras, J., Valdez-Salas, B., Ariza-Aguilar, L. E., Baltazar, M., Nieves, D., Almeraya-Calderón, F., Gaona-Tiburcio, C., Pérez-López, T., López-Vázquez, E., Rodriguez, J., Rebolledo, N., Andrade, C. and Troconis-Rincón, O. (2016). Concrete carbonation in Mexico and Spain: Duracon project, four year evaluation. Key Engineering Materials. 711:12-20. https://doi.org/10.4028/www.scientific.net/KEM.711.12

Nagataki, S., Ohga, H. and Kim, E. K. (1986). Effect of curing conditions on the carbonation of concrete with fly ash and the corrosion of reinforcement in long-term tests. Publication American Concrete Institute. SP 91-24:521-539

Neves, R., Branco, F. and De Brito, J. (2013). Field assessment of the relationship between natural and accelerated concrete carbonation resistance. Cement and Concrete Composites. 41:9-15. https://doi.org/10.1016/j.cemconcomp.2013.04.006

Papadakis, V. G., Fardis, M. N. and Vayenas, C. G. (1992). Effect of composition, environmental factors and cement-lime mortar coating on concrete carbonation. Materials and Structures. 25:293-304 https://doi.org/10.1007/BF02472670

Parrott, L. . (1994). Design for avoiding damage due to carbonation-induced corrosion. International Congress on Durability of Concrete ACI. SP 145-15:283-298.

Parrott, L. J. and Killoh, D. C. (1989). Carbonation in a 36 year old, in-situ concrete. Cement and Concrete Research. 19:649-656 https://doi.org/10.1016/0008-8846(89)90017-3

Possan, E., Andrade, J. J. O., Dal Molin, D. C. C. and Ribeiro, J. L. D. (2021). Model to Estimate Concrete Carbonation Depth and Service Life Prediction. In: "Hygrothermal Behaviour and Building Pathologies". Springer, Porto, Portugal, pp. 67-97. https://doi.org/10.1007/978-3030-50998-9_4

Rabehi, M., Mezghiche, B. and Guettala, S. (2013). Correlation between initial absorption of the cover concrete, the compressive strength and carbonation depth. Construction and Building Materials. 45:123-129. https://doi.org/10.1016/j.conbuildmat.2013.03.074

Ribeiro, A. B., Santos, T. and Gonçalves, A. (2018). Performance of concrete exposed to natural carbonation: Use of the k-value concept. Construction and Building Materials. 175:360-370. https://doi.org/10.1016/j.conbuildmat.2018.04.206

Sanjuán, M. A., Andrade, C. and Cheyrezy, M. (2003). Concrete carbonation tests in natural and 
accelerated conditions. Advances in Cement Research. 15(4):171-180. https://doi.org/10.1680/adcr.2003.15.4.171

Tuutti, K. (1982). Corrosion of steel in concrete, Report 4.82. Swedish Cement and Concrete Research Institute (CBI). Estocolmo, Suecia, p.35.

Van Den Heede, P. and De Belie, N. (2014). A service life based global warming potential for high-volume fly ash concrete exposed to carbonation. Construction and Building Materials. 55:173-183. https://doi.org/10.1016/j.conbuildmat.2014.01.033

Visser, J. H. M. (2014). Influence of the carbon dioxide concentration on the resistance to carbonation of concrete. Construction and Building Materials. 67A:8-13 https://doi.org/10.1016/j.conbuildmat.2013.11.005

von Greve-Dierfeld, S., Lothenbach, B., Vollpracht, A., Wu, B., Huet, B., Andrade, C., Medina, C., Thiel, C., Gruyaert, E., Vanoutrive, H., Saéz del Bosque, I. F., Ignjatovic, I., Elsen, J., Provis, J. L., Scrivener, K., Thienel, K. C., Sideris, K., Zajac, M., Alderete, N., ... De Belie, N. (2020). Understanding the carbonation of concrete with supplementary cementitious materials: a critical review by RILEM TC 281-CCC. Materials and Structures/Materiaux et Constructions. 53:136 https://doi.org/10.1617/s11527-020-01558-w

Wierig, H. (1984). Longtime studies on the carbonation of concrete under normal outdoor exposure. Proceedings of the RILEM, Hannover University, pp. 239-249.

Yoon, I. S., Çopuroğlu, O. and Park, K. B. (2007). Effect of global climatic change on carbonation progress of concrete. Atmospheric Environment. 41:7274-7285 https://doi.org/10.1016/j.atmosenv.2007.05.028

Zega, C. J., Etcheverry, J. M. and Villagrán-Zaccardi, Y. A. (2019). Natural Carbonation of Multiplely Recycled Aggregate Concrete. Proceedings of the International Workshop CO2 Storage in Concrete CO2STO2019, pp. 117-123. 